\title{
Elevation-Dependent Influence of Snow Accumulation on
}

\section{Forest Greening}

by

Ernesto Trujillo ${ }^{1,2}$, Noah P. Molotch ${ }^{1,3,4}$, Michael L. Goulden ${ }^{5}$, Anne E. Kelly ${ }^{5}$, and Roger

C. Bales ${ }^{6}$

1 Institute of Arctic and Alpine Research, University of Colorado, Boulder, CO 80309-0450, USA

2 Laboratory of Cryospheric Sciences, School of Architecture, Civil and Environmental Engineering, Ecole Polytechnique Fédérale de Lausanne, Lausanne, Switzerland

3 Department of Geography, University of Colorado, Boulder, CO 80309-0450, USA

4 Jet Propulsion Laboratory, California Institute of Technology, Pasadena, CA 91109-8099, USA

5. Department of Earth System Science, University of California, Irvine, CA 92697-3100, USA

6. Sierra Nevada Research Institute, University of California, Merced, CA 95343, USA 


\section{Description of Predominant Land Cover Types}

The predominant land cover types in the study region are woodland $(41 \%)$, evergreen needleleaf forest (28\%), wooded grassland (13\%), and grassland (10\%). Woodlands refers to lands with herbaceous or woody understories and tree canopy cover between $40 \%$ and $60 \%$, trees exceed $5 \mathrm{~m}$ in height and can be either evergreen or deciduous; evergreen needleleaf forests refers to lands dominated by trees with a per cent canopy cover $>60 \%$ and height exceeding $5 \mathrm{~m}$, and canopy is never without green foliage; wooded grasslands refers to lands with herbaceous or woody understories and tree canopy cover between $10 \%$ and $40 \%$, trees exceed $5 \mathrm{~m}$ in height and can be either evergreen or deciduous; grasslands refers to lands with continuous herbaceous cover and $<10 \%$ tree or shrub canopy cover. 


\section{Spatial Features of the GIMMS NDVI Product in the Sierra Nevada Region}

The following analysis has the objective of illustrating the capability of the GIMMS NDVI 8$\mathrm{km}$ dataset to capture the spatial and elevational structure of NDVI during the growing season in the Sierra Nevada region. In the analysis, we perform a comparison between the GIMMS NDVI product, and the Terra MODIS 250-m product (MOD13Q1, https://lpdaac.usgs.gov/lpdaac/products/) aggregated by average to match the 8-km scale of the GIMMS NDVI grid. Quality Assurance (QA) of the MODIS NDVI values was accounted for using MOD13Q1 Band 2, which contains detailed QA information (16-bit binary, see Band 2 description at https://lpdaac.usgs.gov, under the MOD13Q1 information). Only values denominated as "good quality" (Bits 0-1 =00) were used. This comparison affords an assessment of the GIMMS product relative to a data set derived at significantly higher spatial and spectral resolution (i.e., MODIS data). We use the concurrent composites of both products during the biweekly periods in July and August of each year, for the period 20002006 ( 4 per year, for 7 years, for a total of 28 direct comparisons). $\mathrm{R}^{2}$ values for the pixel-bypixel comparison of the two products for all concurrent dates have a mean of 0.72 ; all statistically significant with p-values $<0.01$. Four sample scatter plots for the second biweekly period in the month of July are shown in Figure S1; all comparisons show a similar pattern. Complementary, the elevational features of both products were compared by obtaining the mean NDVI for 250-m elevation bands from both products at the 8-km scale (four sample plots are shown in Figure S2). The average $\mathrm{R}^{2}$ for all of the 28 comparisons is 0.97 , all statistically significant with p-values $<<0.01$. All the scatter plots show a pattern around the 1:1 line, similar to that of the plots in Figure S2. The results from these comparisons indicate that the GIMMS NDVI dataset accurately captures the spatial and elevational features of growing season NDVI as observed from MODIS. Hence, the product is adequate to infer relationships between growing season NDVI and snowpack accumulation across the region, and across elevations. Other studies have also demonstrated strong agreement between AVHRR NDVI and other NDVI products like MODIS NDVI (MOD13) when aggregated at the same grid scales for several land cover types, including deciduous and evergreen forest ${ }^{28-30}$. 


\section{Assessment of the Sub-grid Scale Distribution of the MOD13Q1 NDVI within the Overlying GIMMS 8-km grid-cells in the Sierra Nevada Region}

Spatial variability in snow and NDVI patterns at scales finer than 8-km (e.g. associated with slope and aspect) may also contribute to the broader scale relationships resolved under this study. In order to evaluate the effects of this finer-scale variability on the GIMMS NDVI-SWE relationships we evaluated the empirical density and cumulative distribution functions within each of the 8-km GIMMS grid-cells for the four biweekly periods (JulyAugust) for the years 2000-2006 (7 years, 4 biweekly periods, a total of 28 periods). The analysis was performed for all GIMMS grid-cells with a $250-\mathrm{m}$ cell count of at least $75 \%$ of the maximum number of grid-cells (the maximum number of grid-cells is $32 \times 32=1024$ ). Grid-cell counts can be reduced for the cells along the border of the study area or due to water bodies and other unfavorable Quality Assurance flags. Only values denominated as "good quality" (QA Band 2, Bits 0-1 =00) were used. The sub-grid distributions were analyzed using standardized NDVI values (mean of zero and standard deviation of one), such that the distributions can be effectively compared to the standard normal distribution. This is the reason why the range of the $\mathrm{x}$-axis in the figures to follow covers a range between -5 to 5 (Figures S3, S4 and S6).

The sub-grid distributions of the MOD13Q1 NDVI show a uni-modal pattern with little to no bias around the mean (Figure S3). The majority of the empirical density and cumulative distributions (Figures S3 and S4, respectively) are very close to the theoretical standard normal distribution (red line in Figures S3 and S4). The skewness coefficients for these distributions are concentrated around a value very close to zero (Figure S5), with a slightly negative mean (red vertical lines).

We also obtained the distributions of MOD13Q1 NDVI within six elevation bands of $250-\mathrm{m}$ between $2000 \mathrm{~m}$ and $3500 \mathrm{~m}$ (Figure S6). Similarly, these empirical distributions of NDVI are very close to a normal distribution, although the distributions within some of the elevation bands show a more pronounced peak and a slightly negative skewness.

In summary, the combined results here demonstrate that: (a) the 8-km GIMMS product is able to reproduce both the regional and elevational variations in NDVI, (b) the sub-grid scale distributions of MOD13Q1 NDVI closely resemble a normal distribution within the overlying GIMMS 8-km grid cells, with little to no skewness around the mean and a uni-modal pattern, and (c) the distributions of MOD13Q1 NDVI within elevation bands coincident with those analyzed in the study are uni-modal and closely resemble a normal distribution, which shows that the mean value is representative of the average conditions within the pixels, even more so given the low skewness of the distributions. These combined results support the use of the GIMMS NDVI dataset in the context of this study. 


\section{Representativeness of the Snow Pillow Stations}

To illustrate the capability of the snow station data for capturing the regional snowpack conditions, we performed an analysis of the consistency and reliability of the station data in the Sierra Nevada region. Firstly, we iteratively compared the time series of peak SWE of each individual station to all other stations to determine the consistency of the stations across the Sierra Nevada domain. The correlation values between the individual stations are remarkably high (Figure S7a), with $93 \%$ of the values exceeding 0.6 , while $62 \%$ exceeding 0.8. Secondly, the time series of peak SWE at the individual stations show very strong correspondence to the regional mean (Figure $\mathrm{S} 7 \mathrm{~b}$ ). The majority of the correlation coefficients exceed 0.6 , with only a few values below this threshold. The correspondence between the individual time series and the regional mean exhibits a strong elevational gradient, with correlation values increasing with elevation, particularly at the lower elevation range. These results illustrate that: (a) the time series of peak SWE at each station correlates well with the time series at other stations in the region, and that (b) the time series of peak SWE at each stations is highly correlated with the regional mean peak SWE. Consequently, the station data is adequate to infer regional information about the interannual variability of snow accumulation over the region. 
(a) 2000

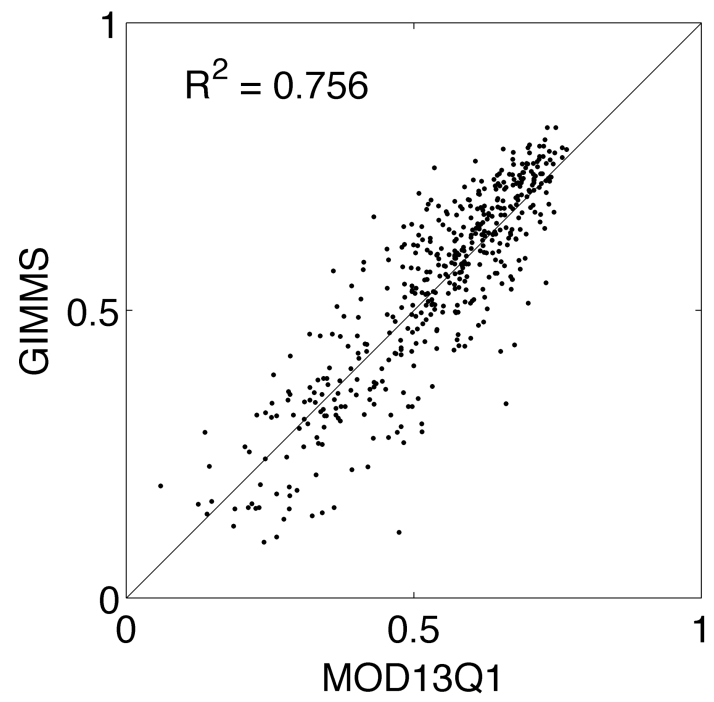

(c) 2004

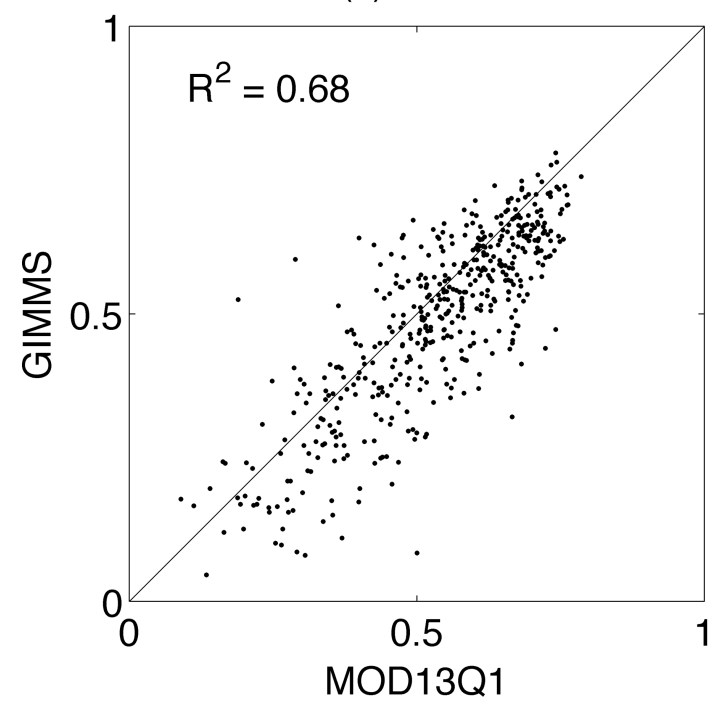

(b) 2002

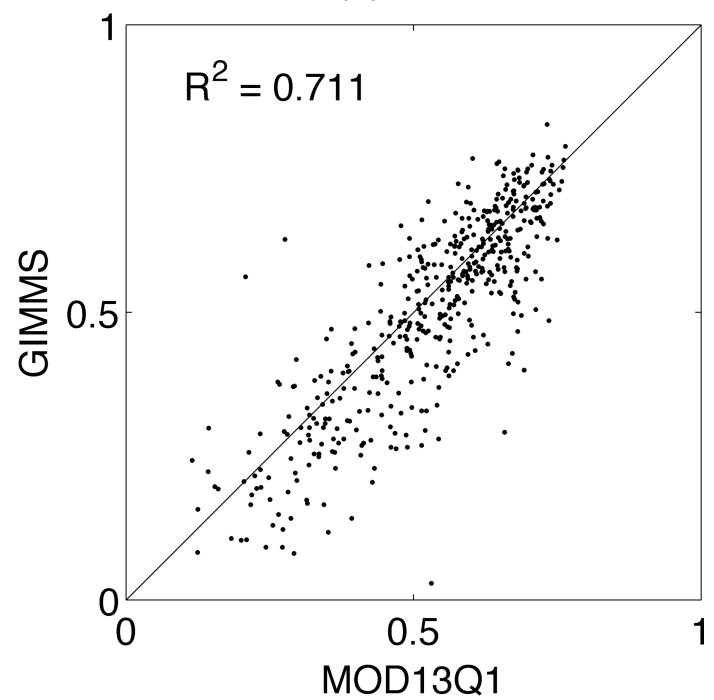

(d) 2006

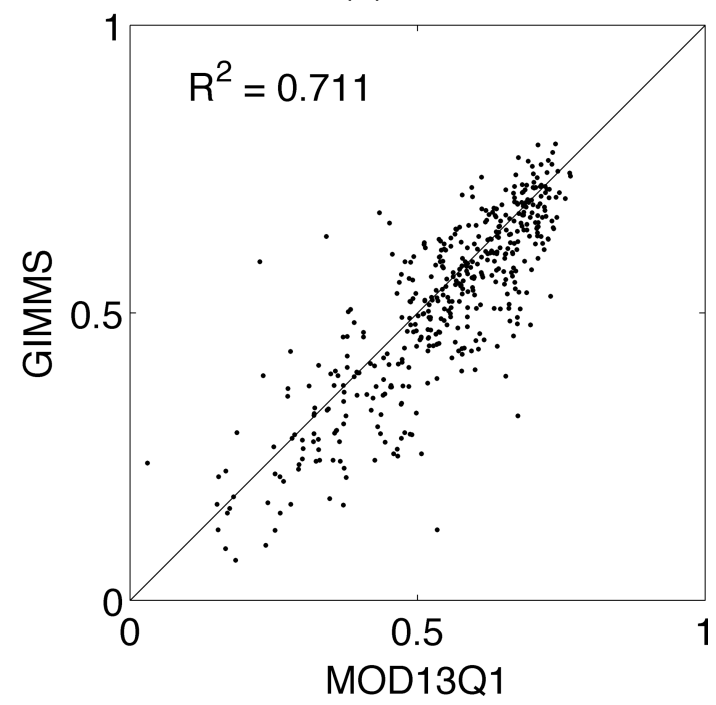

Figure S1. Pixel by pixel comparison of GIMMS and MODIS NDVI. Scatter plots of the pixel values at the 8-km scale from both, GIMMS and MOD13Q1 NDVI products, for the second biweekly period in July for four sample years ( $\mathrm{p}$-values $<<0.01$ ). 
(a) July-a

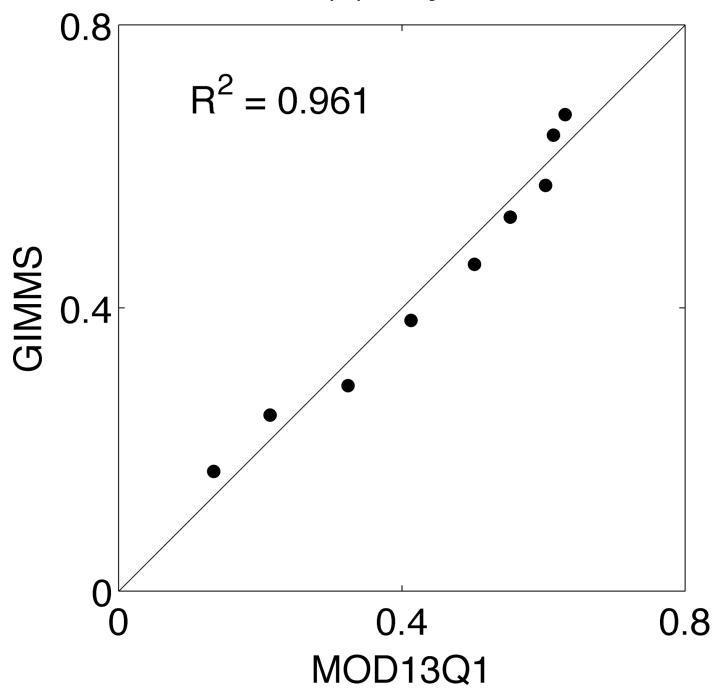

(c) August-a

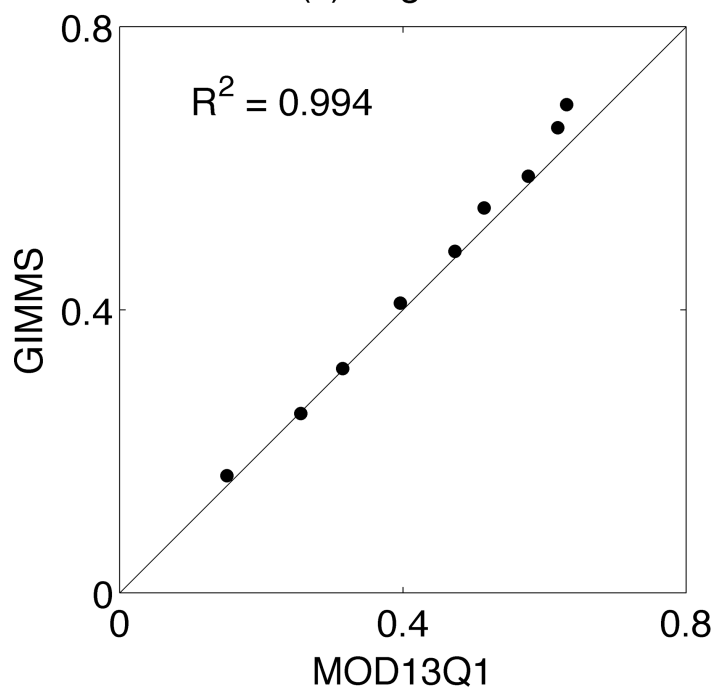

(b) July-b

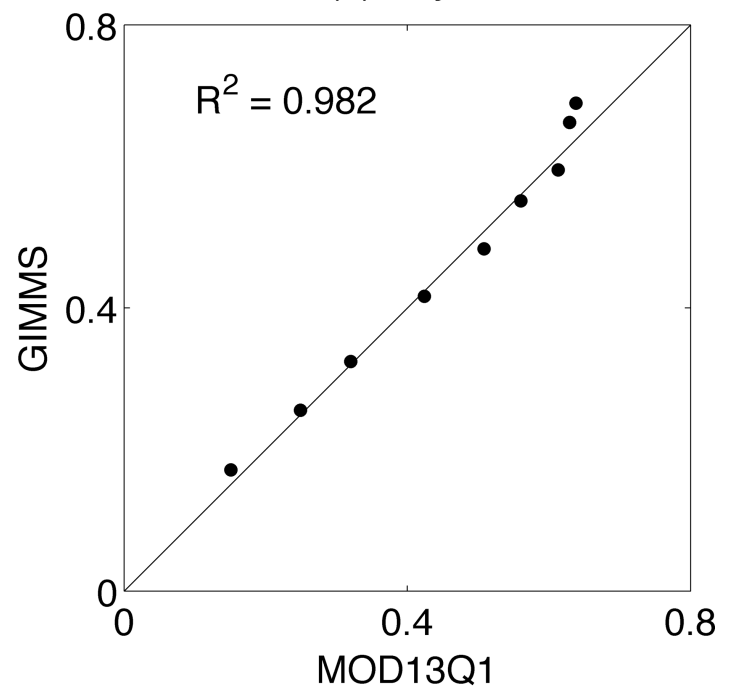

(d) August-b

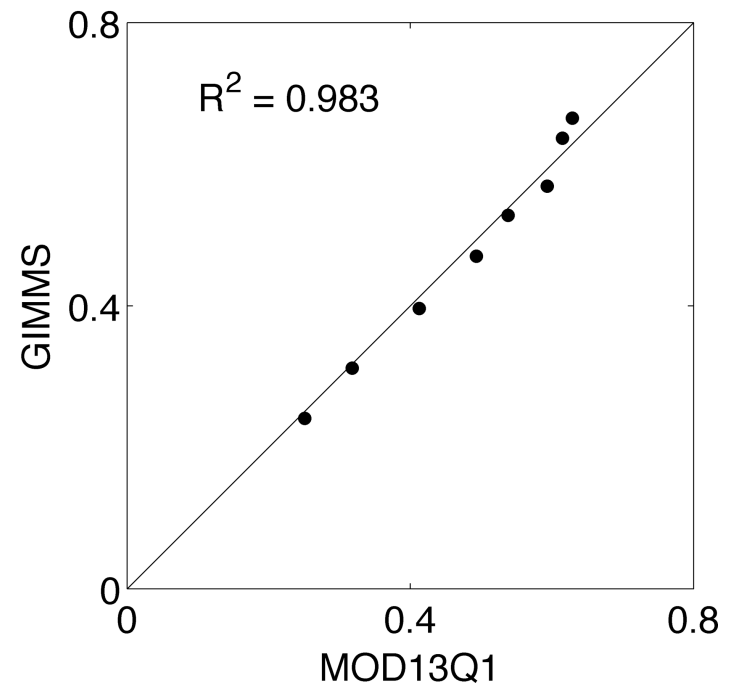

Figure S2. Comparison of elevational patterns from GIMMS and MODIS NDVI. Scatter plots of mean NDVI from both GIMMS and MOD13Q1 products for 250-m elevation bands across the Sierra Nevada region. The four panels correspond to the biweekly periods of the year 2000 (p-values $<<0.01)$. 
(a) 2000

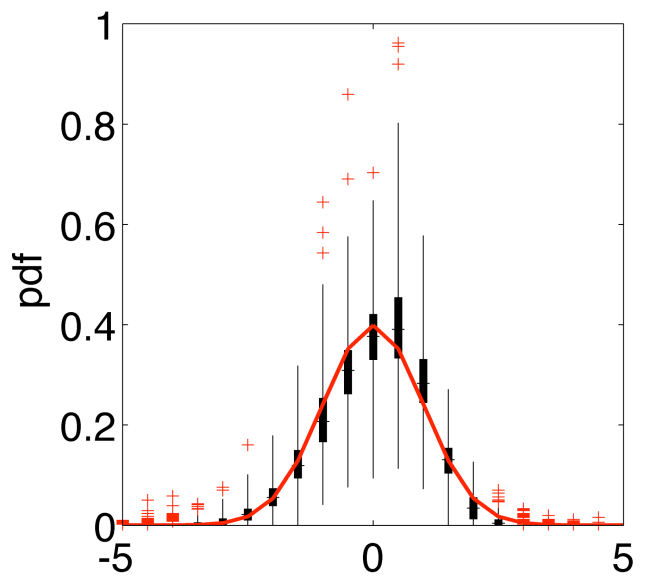

(c) 2004

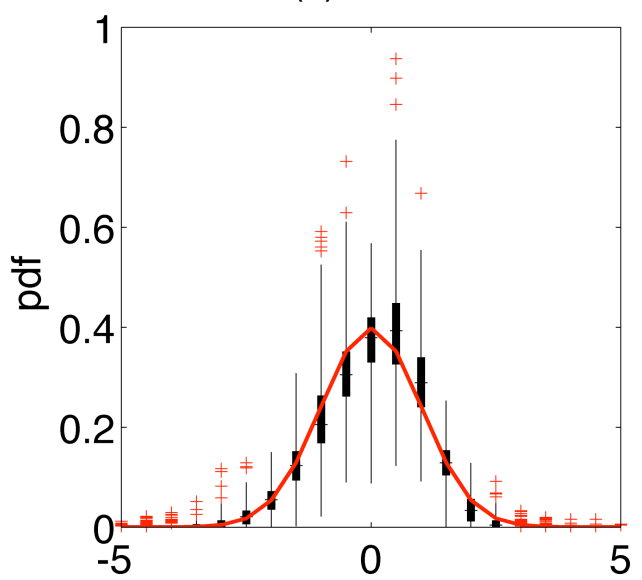

(b) 2002

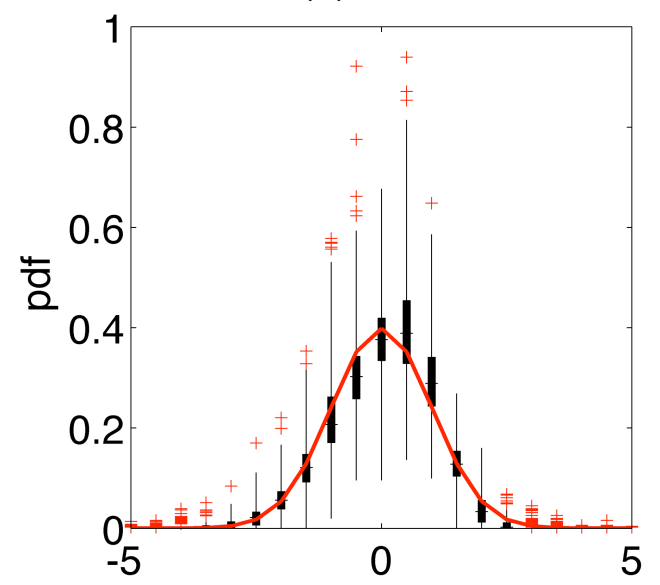

(d) 2006

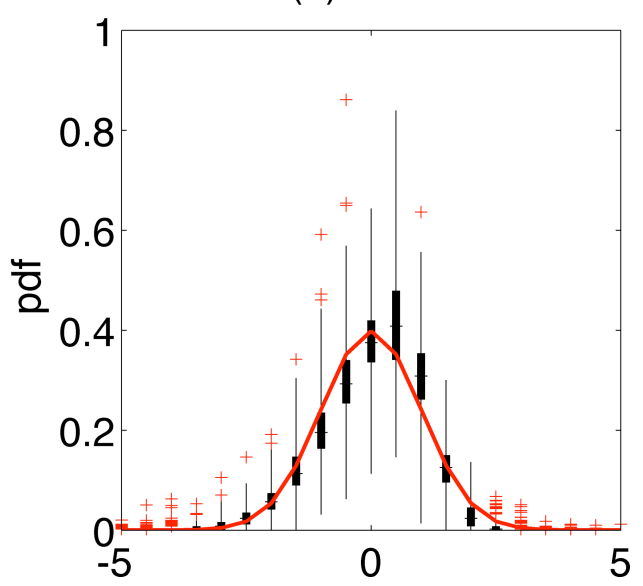

Figure S3. Assessment of the sub-pixel distribution of NDVI. Box plots of the standardized empirical probability density functions (epdf's) of the 250-m MODIS NDVI values within each of the overlaying 8-km pixels. Each panel corresponds to the second biweekly period of July for four years, although similar results were observed for all the periods analyzed. The black inside boxes group $50 \%$ of the distributions while the red plus signs mark outlier values. The standard normal distribution is shown in red. 
(a) 2000

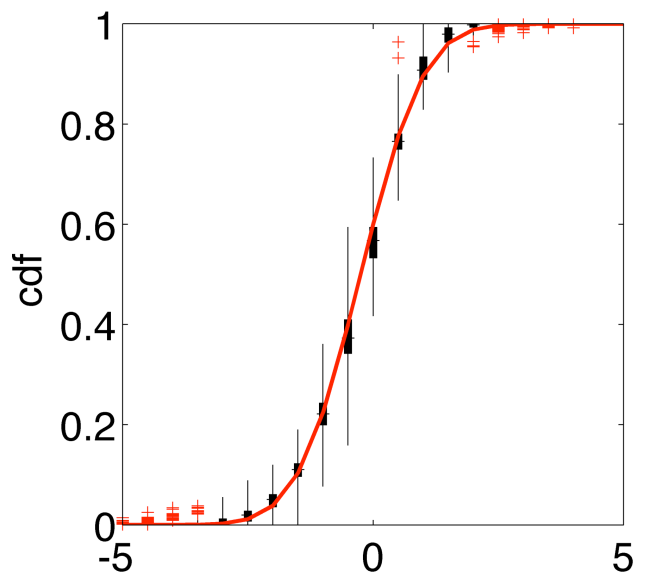

(c) 2004

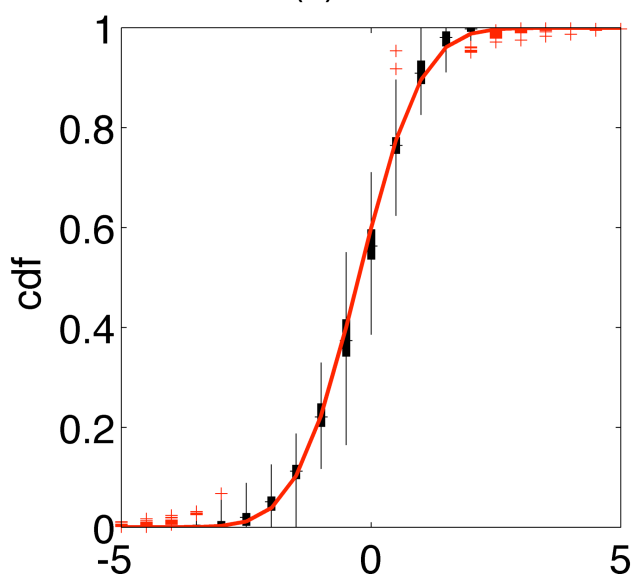

(b) 2002

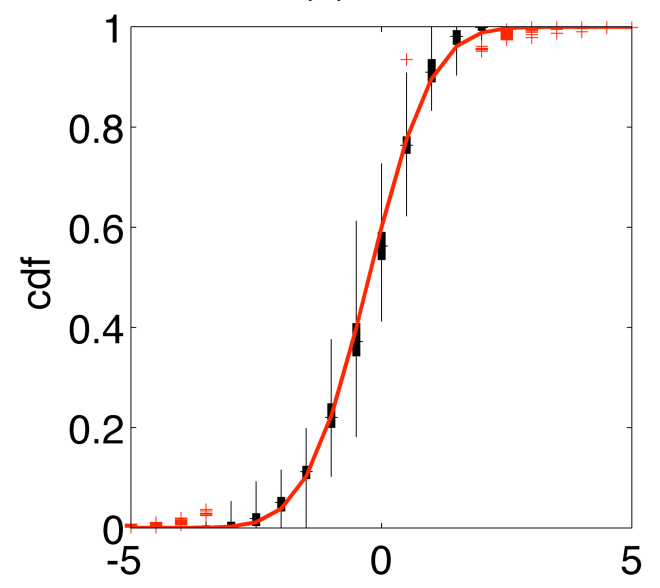

(d) 2006

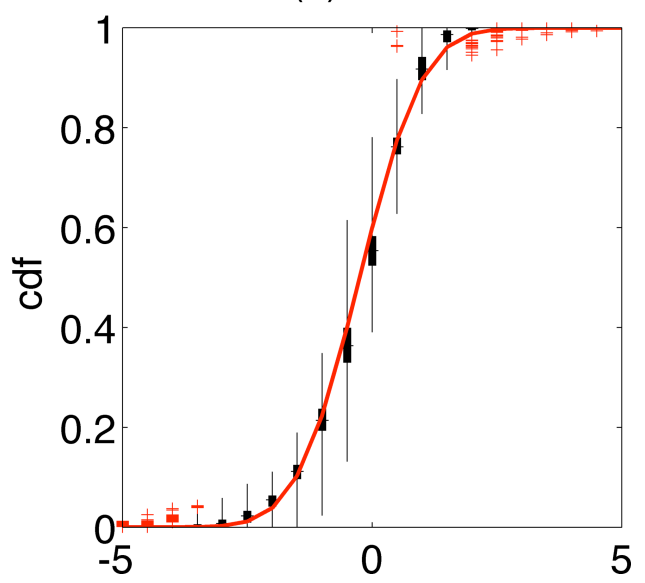

Figure S4. Assessment of the sub-pixel cumulative distribution of NDVI. Box plots of the empirical cumulative distribution functions of MODIS NDVI obtained from the epdf's in Figure S3. Each panel corresponds to the second bi-weekly period of July for four years, although similar results were observed for all the periods analyzed. The black inside boxes group $50 \%$ of the distributions while the red plus signs mark outlier values. The standard normal cumulative distribution is shown in red. 
(a) 2000

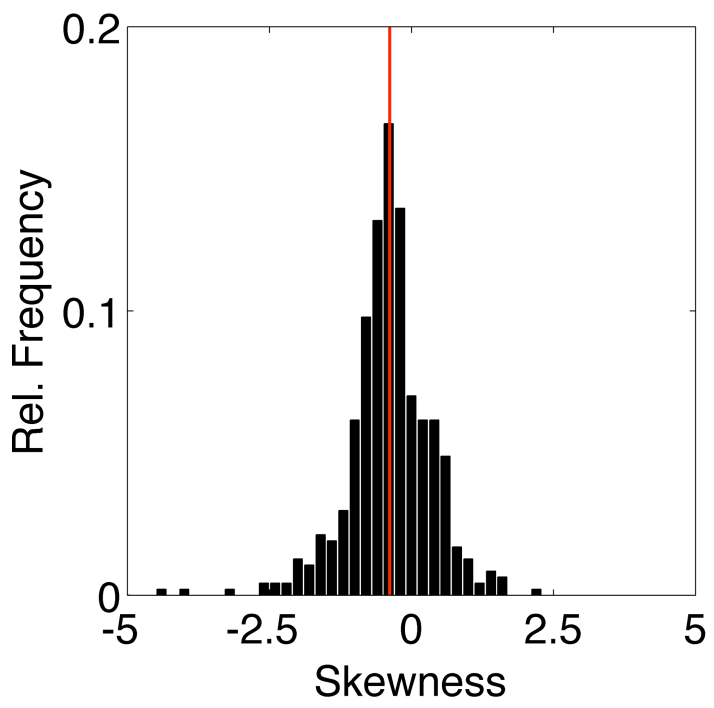

(c) 2004

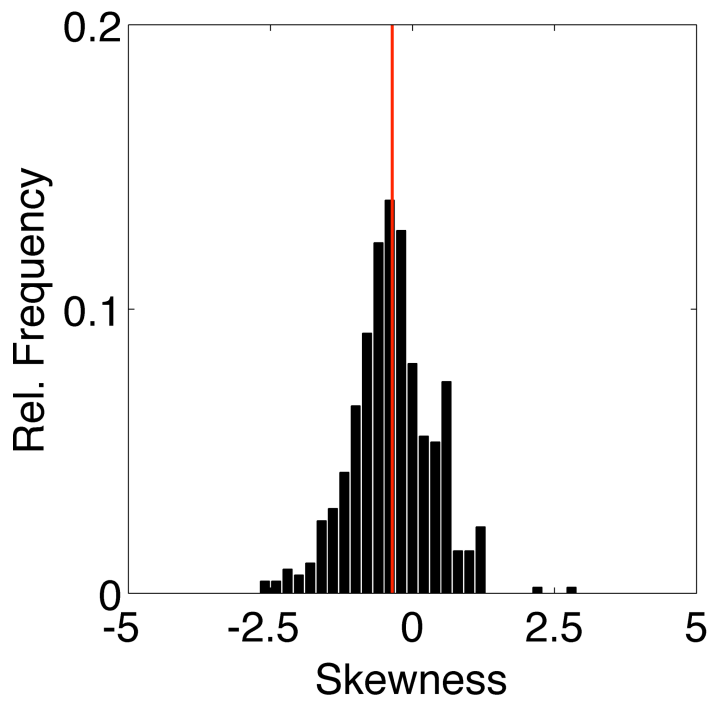

(b) 2002

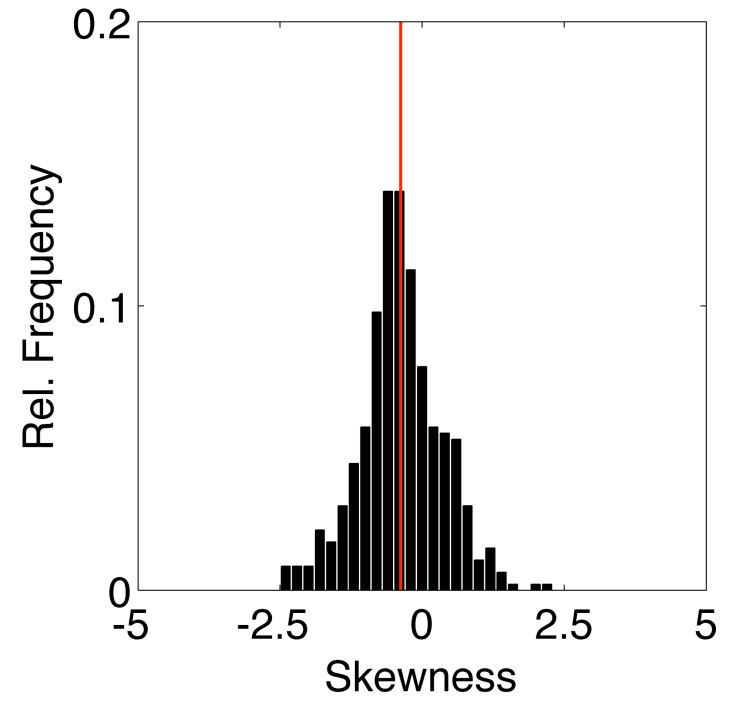

(d) 2006

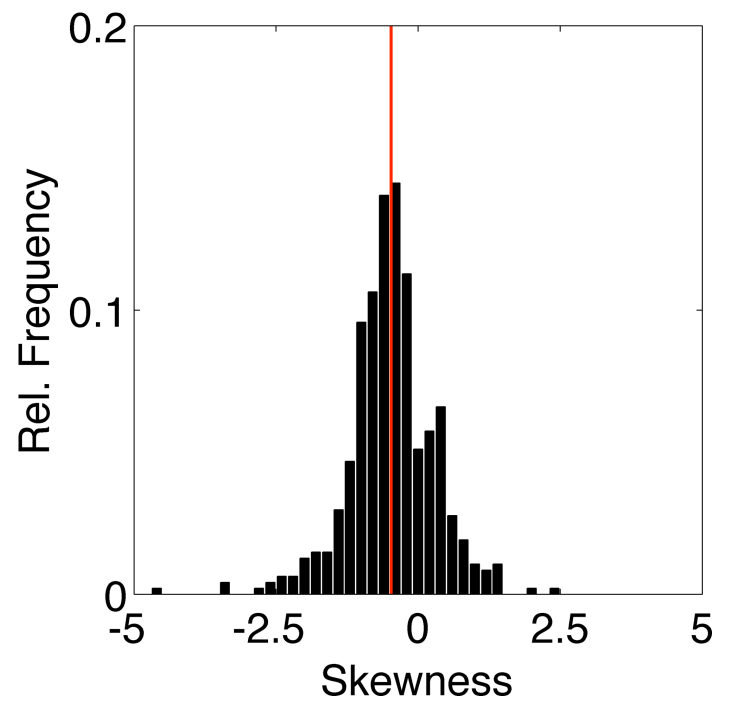

Figure S5. Assessment of the skewness of NDVI within 8-km grid-cells. Histograms of the skewness of the MODIS NDVI distributions in Figure S3. The majority of the values are concentrated around a value near zero, with a slight left skew bias. The red line is the mean skewness. 
(a) 2000

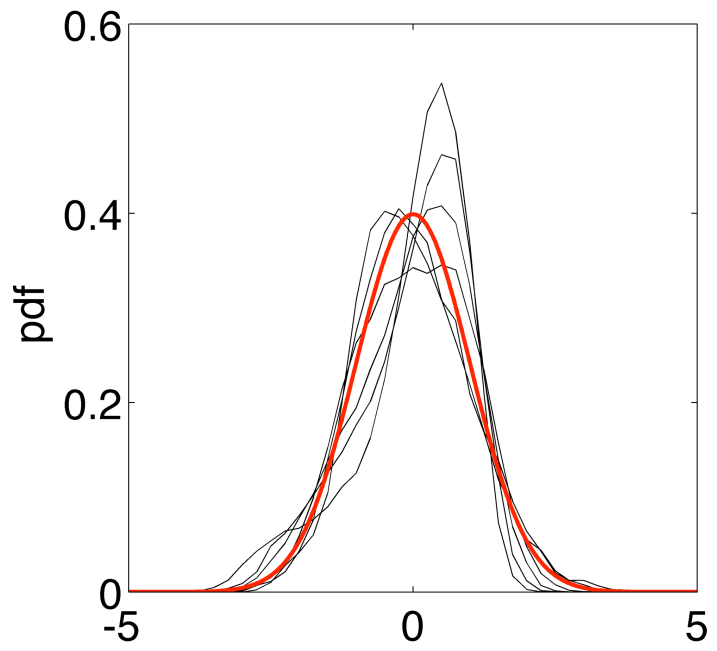

(c) 2004

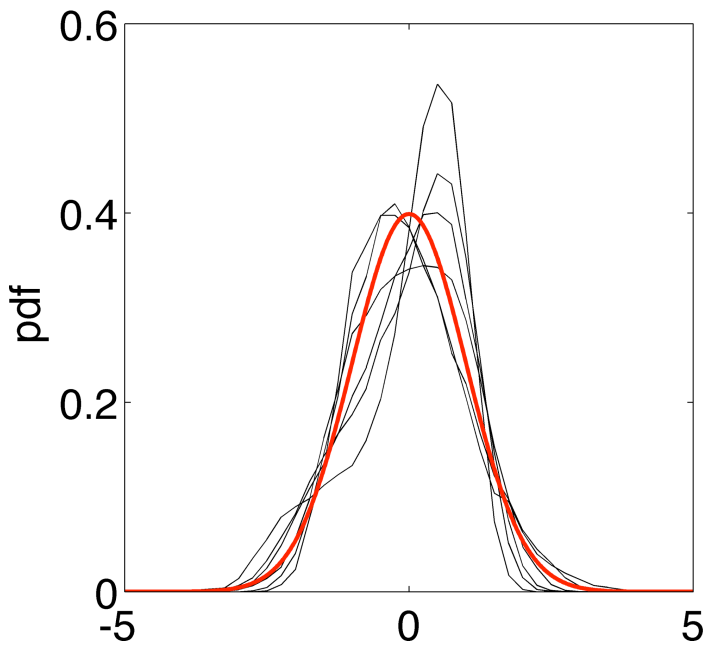

(b) 2002

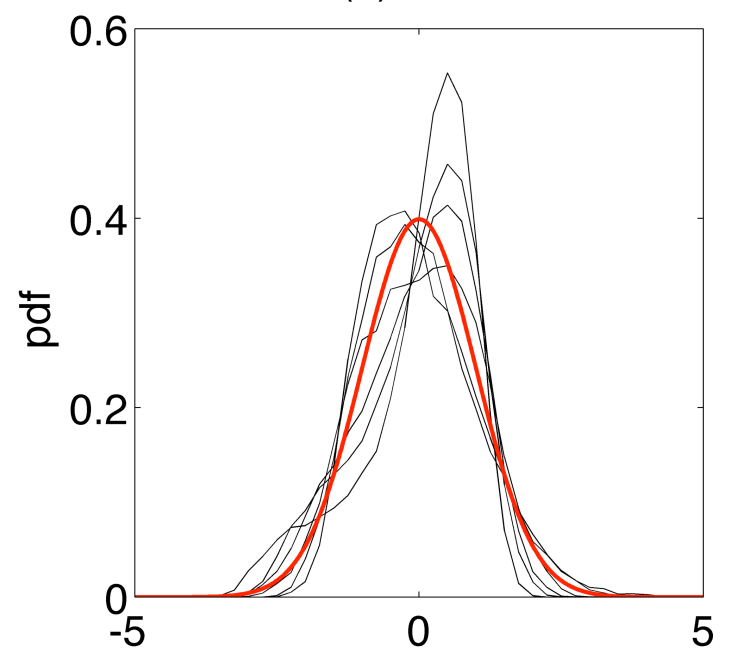

(d) 2006

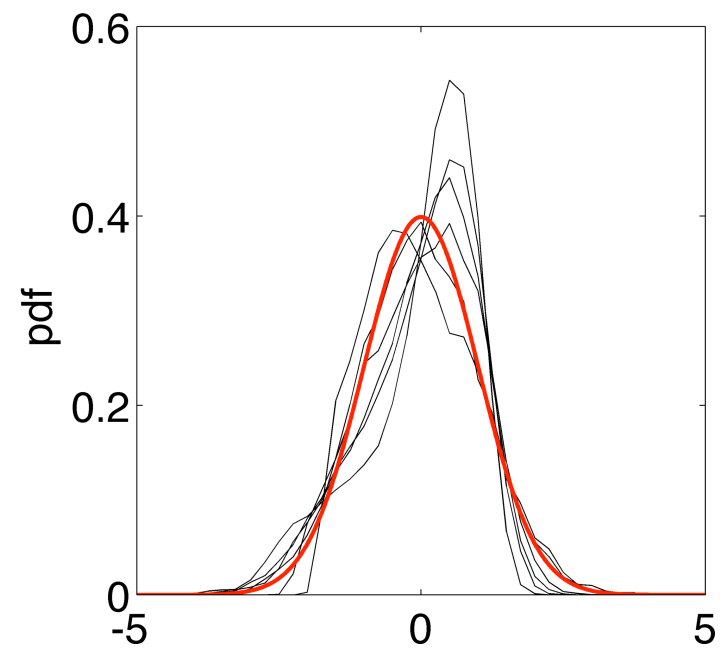

Figure S6. Assessment of the distribution of NDVI within elevation bands. Standardized empirical density functions for the MODIS NDVI values within elevation bands of $250-\mathrm{m}$ (along the vertical scale) for a range between $2000 \mathrm{~m}$ and $3500 \mathrm{~m}$ (6 bands). Elevations form a digital elevation model at a $250-\mathrm{m}$ horizontal scale were used to define the elevation bands. 
(a)

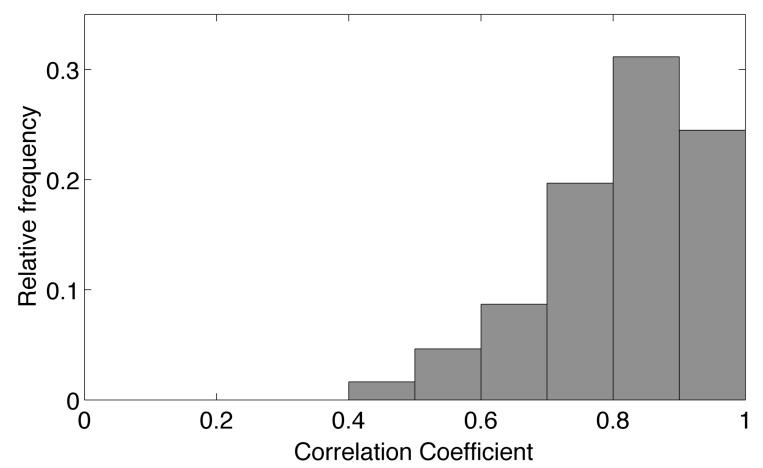

(b)

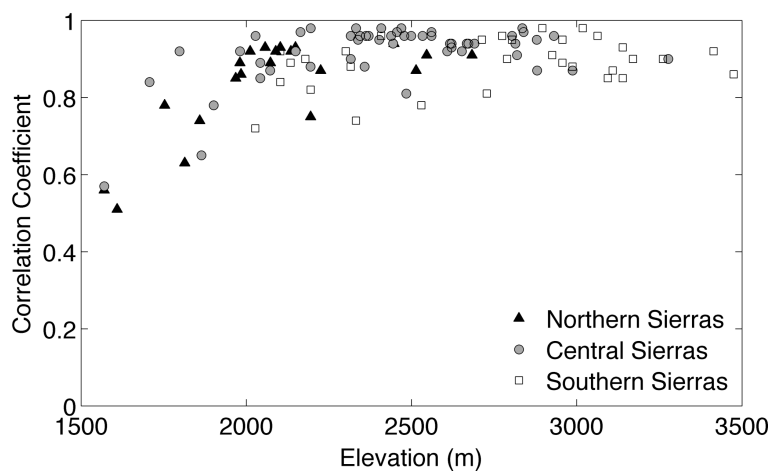

Figure S7. Regional representativeness of the snow pillow stations within the Sierra Nevada Mountains. (a) Histogram (relative frequency) of the correlation coefficient values relating peak SWE at each snow station to all other individual snow stations. (b) Correlation coefficients between the time series of the individual stations and the mean series in the Sierra Nevada region. 